Georgian Mathematical Journal

Volume 11 (2004), Number 1, 125-135

\title{
THE BELLMAN EQUATION RELATED TO THE MINIMAL ENTROPY MARTINGALE MEASURE
}

\author{
M. MANIA, M. SANTACROCE, AND R. TEVZADZE
}

\begin{abstract}
We derive a backward stochastic differential equation and a Bellman equation characterizing the minimal entropy martingale measure for market models, where asset prices are driven by Markov diffusion processes. A relation between these equations is established.
\end{abstract}

2000 Mathematics Subject Classification: 91B28, 60H30, 90C39.

Key words and phrases: Minimal entropy martingale measure, backward stochastic differential equation, Bellman equation, incomplete market, stochastic volatility model.

\section{INTRODUCTION}

The problem of finding the minimal entropy martingale measure is dual to the problem of maximizing the expected exponential utility from terminal wealth and is used to determine an optimal strategy by exponential hedging of contingent claims (see [4] and [15]). The minimal entropy martingale measure is defined as the martingale measure minimizing its relative entropy with respect to the basic probability measure. It is known (see [7] and [13]), that, for a locally bounded semimartingale, the minimal entropy martingale measure always exists, is unique and, if there is a martingale measure with finite relative entropy, then, it is equivalent to the basic measure. Having in mind its role in the contingent claims pricing, we focus our attention on finding the minimal entropy martingale measure and on studying its structure.

This paper specializes the results obtained by the authors in [12] in a continuous semimartingale setting to the case where the price process is a Markov diffusion process. In Section 2 we formulate, in a form suitable for diffusion processes, the basic statement of [12] which characterizes the minimal entropy martingale measure in terms of the corresponding value process shown to be the unique solution of a backward stochastic differential equation (BSDE). In fact, here we aim at establishing a connection between the semimartingale backward equation for the value process and the classical Bellman equation for the value function related to the same problem in the case of Markov diffusion processes. Since in this case the value process can be represented as a space-transformation of an asset price process by means of the value function, the problem is to derive the differentiability properties of the value function from the fact that the value process satisfies the corresponding BSDE. The key role connecting the above mentioned equations is played by the statements describing all invariant space-transformations of diffusion processes, studied in [2] and adapted here to 
financial market models. This approach enables us to prove that there exists a solution (in a certain sense) of the Bellman equation and that this solution is differentiable (in a generalized sense) under mild assumptions on the model coefficients. Although, in our case, the generalized derivative at $t$ and second order generalized derivatives at $x$ do not exist separately in general (we prove existence of a generalized $L$-operator), these derivatives do not participate in the construction of the optimal martingale measure and the optimal strategy is explicitly given in terms of first order derivatives of the value function. It is worth noticing that in the theory of viscosity solutions, usually applied to such problems (see, e.g., [5]), the differentiability of the value function goes in general beyond the reach of the method.

Moreover, we study two specific extreme cases (considered in [14], [11], [1] in relation to the variance-optimal martingale measures), where the Bellman equation admits an explicit solution and provides an explicit construction of the minimal entropy martingale measure.

\section{The Market Model Driven by an Itô Process}

We consider a diffusion model for the financial market as in [9] and [11]. $W=\left(W^{1}, \ldots, W^{n}\right)$ is an $n$-dimensional standard Brownian motion defined on a complete probability space $(\Omega, \mathcal{F}, P)$ equipped with the $P$-augmentated filtration generated by $W, F=\left(F_{t}, t \in[0, T]\right), \mathcal{F}=F_{T} . W^{l}=\left(W^{1}, \ldots, W^{d}\right)$ and $W^{\perp}=\left(W^{d+1}, \ldots, W^{n}\right)$ are $d$ - and $(n-d)$-dimensional Brownian motion, respectively.

Assume that there are $d$ risky assets (stocks) and a bond traded on the market. For simplicity, the bond price is assumed to be 1 at all times and the stock price dynamics is given by

$$
d X_{t}=\operatorname{diag}\left(X_{t}\right)\left(\mu_{t} d t+\sigma_{t} d W_{t}^{l}\right), \quad t \in[0, T],
$$

where $\operatorname{diag}(X)$ denotes the diagonal $d \times d$-matrix with diagonal elements $\left(X^{1}, \ldots, X^{d}\right)$.

The market coefficients: the $d$-dimensional vector process $\mu$ of stock appreciation rates and the $d \times d$ volatility matrix $\sigma$ are progressively measurable with respect to $F$. We also require that for any $t \in[0, T]$ the volatility matrix be nonsingular almost surely. We take $n>d$ so that there are more sources of uncertainty than the stocks available for trading and the market is incomplete.

Let us denote by $\theta$ the market price of risk $\sigma^{-1} \mu$ and assume that the mean variance tradeoff $\int_{0}^{T}\left\|\theta_{s}\right\|^{2} d s$ is finite a.s.

Let us call $\mathcal{M}^{e}$ the set of equivalent martingale measures of $X$, i.e., the set of measures equivalent to $P$ under which $X$ is a local martingale. Denoting by $\mathcal{K}(\sigma)$ the class of $F$-predictable $R^{n-d}$ valued processes $\nu$ such that $\int_{0}^{T}\left\|\nu_{t}\right\|^{2} d t<$ $\infty$ a.s., by the integral representation property, the density of any martingale measure $P^{\nu}$ is expressed as an exponential martingale

$$
Z_{t}^{\nu}=\mathcal{E}_{t}\left(-\int_{0} \theta_{s}^{\prime} d W_{s}^{l}+\int_{0} \nu_{s}^{\prime} d W_{s}^{\perp}\right), \quad t \in[0, T],
$$


for some $\nu \in \mathcal{K}(\sigma)$ (see [16]). Let

$$
\mathcal{K}_{\mathcal{E} n t}(\sigma)=\left\{\nu \in \mathcal{K}(\sigma): E Z_{T}^{\nu}=1, E Z_{T}^{\nu} \ln Z_{T}^{\nu}<\infty\right\}
$$

and consider the subclass of equivalent martingale measures $\mathcal{M}_{\mathcal{E} n t}^{e}$ defined by

$$
\mathcal{M}_{\mathcal{E} n t}^{e}=\left\{P^{\nu}: d P^{\nu} / d P=Z_{T}^{\nu}, \nu \in \mathcal{K}_{\mathcal{E} n t}(\sigma)\right\}
$$

We can now express the minimal entropy martingale measure as a solution of the following optimization problem

$$
\inf _{\nu \in \mathcal{K}_{\mathcal{E} n t}(\sigma)} E Z_{T}^{\nu} \ln Z_{T}^{\nu}
$$

As shown in [7], if $\mathcal{M}_{\mathcal{E} n t}^{e}$ is not empty (or equivalently, if $\mathcal{K}_{\mathcal{E} n t}(\sigma) \neq \emptyset$ ), then there exists a unique solution of (2.4) which gives a martingale measure equivalent to $P$.

Let us assume that

A) there exists a martingale measure, $P^{\nu}$, that satisfies the reverse Hölder $R_{\mathcal{E} n t}$-inequality, i.e. the corresponding density process $Z^{\nu}$ is a strictly positive uniformly integrable martingale and there is a constant $C$ such that, for every stopping time $\tau$,

$$
E\left(\frac{Z_{T}^{\nu}}{Z_{\tau}^{\nu}} \ln \frac{Z_{T}^{\nu}}{Z_{\tau}^{\nu}} \mid F_{\tau}\right) \leq C
$$

Remark. Note that condition A) is satisfied if the mean variance tradeoff is bounded.

According to Corollary 3.2 from [12], problem (2.4) is equivalent to

$$
\frac{1}{2} \inf _{\nu \in \mathcal{K}_{\mathcal{E} n t}(\sigma)} E^{\nu} \int_{0}^{T}\left(\left\|\theta_{s}\right\|^{2}+\left\|\nu_{s}\right\|^{2}\right) d s
$$

and the corresponding value process takes the form

$$
V_{t}=\frac{1}{2} \underset{\nu \in \mathcal{K}_{\mathcal{E} n t}(\sigma)}{\operatorname{essinf}} E^{\nu}\left(\int_{t}^{T}\left(\left\|\theta_{s}\right\|^{2}+\left\|\nu_{s}\right\|^{2}\right) d s \mid F_{t}\right) .
$$

The next statement proved in [12] is adapted here to diffusion processes.

Theorem 1. Let condition A) be satisfied. Then the value process $V$ is the unique bounded positive solution of the BSDE

$$
V_{t}=V_{0}-\int_{0}^{t}\left[\frac{1}{2}\left\|\theta_{s}\right\|^{2}-\theta_{s}^{\prime} \varphi_{s}-\frac{1}{2}\left\|\varphi_{s}^{\perp}\right\|^{2}\right] d s+\int_{0}^{t} \varphi_{s}^{\prime} d W_{s}^{l}+\int_{0}^{t} \varphi_{s}^{\perp^{\prime}} d W_{s}^{\perp}, V_{T}=0
$$

and the martingale part of the value process belongs to the class BMO (for $B M O$-martingales refer to $[10])$.

Moreover, $\nu^{*}$ is optimal if and only if

$$
\nu_{t}^{*}=-\varphi_{t}^{\perp} \quad d t \times d P \text {-a.e. },
$$


i.e., the density of the minimal entropy martingale measure is of the form

$$
Z_{T}^{\nu^{*}}=\mathcal{E}_{T}\left(-\int_{0} \theta_{s}^{\prime} d W_{s}^{l}-\int_{0} \varphi_{s}^{\perp^{\prime}} d W_{s}^{\perp}\right) .
$$

Remark. In [12], the unicity of the solution of (2.7) was proved assuming the existence of the minimal martingale measure satisfying the reverse Hölder $R_{\mathcal{E} n t^{-}}$ inequality. But this statement remains true if the reverse Hölder $R_{\mathcal{E} n t}$-inequality is satisfied for some martingale measure.

\section{Stochastic Volatility Models}

Considering a stochastic volatility model similar to [14], we will assume that the dynamics of the assets price process is determined by the following system of stochastic differential equations:

$$
\begin{aligned}
d X_{t} & =\operatorname{diag}\left(X_{t}\right)\left(\mu\left(t, X_{t}, Y_{t}\right) d t+\sigma^{l}\left(t, X_{t}, Y_{t}\right) d W_{t}^{l}\right), \\
d Y_{t} & =b\left(t, X_{t}, Y_{t}\right) d t+\delta\left(t, X_{t}, Y_{t}\right) d W_{t}^{l}+\sigma^{\perp}\left(t, X_{t}, Y_{t}\right) d W_{t}^{\perp} .
\end{aligned}
$$

Assume that

B1) the coefficients $\mu, b, \delta, \sigma^{l}, \sigma^{\perp}$ are measurable and bounded;

B2) the $n \times n-$ matrix function $\sigma \sigma^{\prime}$ is uniformly elliptic, i.e., there is a constant $c>0$ such that

$$
(\sigma(t, x, y) \lambda, \sigma(t, x, y) \lambda) \geq c|\lambda|^{2}
$$

for all $t \in[0, T], x \in R_{+}^{d}, y \in R^{n-d}$ and $\lambda \in R^{n}$, where $\sigma$ is defined by

$$
\sigma(t, x, y)=\left(\begin{array}{ll}
\sigma^{l}(t, x, y) & 0 \\
\delta(t, x, y) & \sigma^{\perp}(t, x, y)
\end{array}\right) .
$$

In addition we assume that

B3) system (3.1), (3.2) admits a unique strong solution.

Let us introduce the value function

$$
\begin{aligned}
& V(t, x, y) \\
& =\frac{1}{2} \inf _{\nu \in \mathcal{K}_{\mathcal{E} n t}^{M}(\sigma)} E^{\nu}\left(\int_{t}^{T}\left(\left\|\theta\left(s, X_{s}, Y_{s}\right)\right\|^{2}+\left\|\nu\left(s, X_{s}, Y_{s}\right)\right\|^{2}\right) d s / X_{t}=x, Y_{t}=y\right),
\end{aligned}
$$

where $\theta=\sigma^{l^{-1}} \mu$ and $\mathcal{K}_{\mathcal{E} n t}^{M}(\sigma)$ is the class of feedback controls from $\mathcal{K}_{\mathcal{E} n t}(\sigma)$, i.e., controls $\nu \in \mathcal{K}_{\mathcal{E} n t}(\sigma)$ expressed in the form $\nu\left(t, X_{t}, Y_{t}\right)$ for some measurable function $\nu(t, x, y), t \in[0, T], x \in R_{+}^{d}, y \in R^{n-d}$.

Remark. Theorem 1 enables us to weaken condition B1), but our aim is now to prove the solvability of the Bellman equation without any regularity assumption on the coefficients. Note also that we need to assume B3) only for the financial interpretation of the model and that for the validity of Theorem 2 (and Proposition A) the existence of a unique weak solution of (3.1) and (3.2), guaranteed by B1) and B2), is sufficient. 
Theorem 2. Let condition B1), B2) and B3) be satisfied. Then the value function $V(t, x, y)$ admits all first order generalized derivatives $V_{x}$ and $V_{y}$, a generalized L-operator $L V$ (in the sense of Definition $\mathrm{A}$ in the Appendix) and is the unique bounded solution of equation

$$
\begin{aligned}
& L V(t, x, y)-\theta^{\prime}(t, x, y) \delta^{\prime}(t, x, y) V_{y}(t, x, y)+V_{y}^{\prime}(t, x, y) b(t, x, y)+\frac{1}{2}\|\theta(t, x, y)\|^{2} \\
& \quad+\inf _{\nu \in R^{n-d}}\left[\frac{1}{2}\|\nu\|^{2}+\nu^{\prime} \sigma^{\perp^{\prime}}(t, x, y) V_{y}(t, x, y)\right]=0 \quad d t d x d y \text {-a.s. }
\end{aligned}
$$

with the boundary condition

$$
V(T, x, y)=0 \text {. }
$$

Moreover, $\nu^{*}=-\sigma^{\perp^{\prime}} V_{y}$ and the density of the minimal entropy martingale measure is of the form

$$
Z_{T}^{*}=\mathcal{E}_{T}\left(-\int_{0} \theta^{\prime}\left(s, X_{s}, Y_{s}\right) d W_{s}^{l}-\int_{0}\left(\sigma^{\perp^{\prime}} V_{y}\right)^{\prime}\left(s, X_{s}, Y_{s}\right) d W_{s}^{\perp}\right) .
$$

Proof. Existence. Since $(X, Y)$ is a Markov process, the feedback controls are sufficient and the value process is expressed by

$$
V_{t}=V\left(t, X_{t}, Y_{t}\right) \text { a.s. }
$$

(one can show this fact, e.g., similarly to [2]).

As the value process satisfies equation (2.7), it is an Itô process. It is bounded because of assumptions B1), B2) and by Theorem 1 its martingale part belongs to the space BMO. Hence the finite variation part of $V_{t}$ has the integrable variation. Thus from (3.5) we have that $V\left(t, X_{t}, Y_{t}\right)$ is an Itô process of the form (4.1) (see the Appendix). Proposition A of the Appendix implies that the function $V(t, x, y)$ admits a generalized $L$-operator, all first order generalized derivatives and can be represented as

$$
\begin{aligned}
& V\left(t, X_{t}, Y_{t}\right)=V_{0}+\int_{0}^{t}\left(V_{x}^{\prime}\left(s, X_{s}, Y_{s}\right) \operatorname{diag}\left(X_{s}\right) \sigma^{l}\left(s, X_{s}, Y_{s}\right)+V_{y}^{\prime}\left(s, X_{s}, Y_{s}\right)\right. \\
\times & \left.\delta\left(s, X_{s}, Y_{s}\right)\right) d W_{s}^{l}+\int_{0}^{t} V_{y}^{\prime}\left(s, X_{s}, Y_{s}\right) \sigma^{\perp}\left(s, X_{s}, Y_{s}\right) d W_{s}^{\perp}+\int_{0}^{t} L V\left(s, X_{s}, Y_{s}\right) d s \\
+ & \int_{0}^{t}\left(V_{x}^{\prime}\left(s, X_{s}, Y_{s}\right) \operatorname{diag}\left(X_{s}\right) \mu\left(s, X_{s}, Y_{s}\right)+V_{y}^{\prime}\left(s, X_{s}, Y_{s}\right) b\left(s, X_{s}, Y_{s}\right)\right) d s,
\end{aligned}
$$

where $L V$ is the generalized $L$-operator defined in the Appendix (Definition A).

On the other hand, the value process is a solution of (2.7) and by the uniqueness of the canonical decomposition of semimartingales, comparing the martingale parts of (3.6) and (2.7), we have that $d t \times d P$-a.e.

$$
\varphi_{t}=\sigma^{l^{\prime}}\left(t, X_{t}, Y_{t}\right) \operatorname{diag}\left(X_{t}\right) V_{x}\left(t, X_{t}, Y_{t}\right)+\delta^{\prime}\left(t, X_{t}, Y_{t}\right) V_{y}\left(t, X_{t}, Y_{t}\right)
$$




$$
\varphi_{t}^{\perp}=\sigma^{\perp^{\prime}}\left(t, X_{t}, Y_{t}\right) V_{y}\left(t, X_{t}, Y_{t}\right) .
$$

Then, equalizing the processes of bounded variation of the same equations, taking into account (3.7) and (3.8), we derive

$$
\begin{aligned}
& \int_{0}^{t}\left[V_{y}^{\prime}\left(s, X_{s}, Y_{s}\right) b\left(s, X_{s}, Y_{s}\right)+\frac{1}{2}\left\|\theta\left(s, X_{s}, Y_{s}\right)\right\|^{2}\right. \\
& -\theta^{\prime}\left(s, X_{s}, Y_{s}\right) \delta^{\prime}\left(s, X_{s}, Y_{s}\right) V_{y}\left(s, X_{s}, Y_{s}\right)+L V\left(s, X_{s}, Y_{s}\right) \\
& \left.+\inf _{\nu \in R^{n-d}}\left(\frac{1}{2}\|\nu\|^{2}+\nu^{\prime} \sigma^{\perp^{\prime}}\left(s, X_{s}, Y_{s}\right) V_{y}\left(s, X_{s}, Y_{s}\right)\right)\right] d s=0 .
\end{aligned}
$$

It follows that $V(t, x, y)$ solves the Bellman equation (3.3).

Unicity. Let $\tilde{V}(t, x, y)$ be a bounded positive solution of (3.3), (3.4), from the class $V^{L}$. Then using the generalized Itô formula (Proposition A of the Appendix) and equation (3.3) we obtain that $\tilde{V}\left(t, X_{t}, Y_{t}\right)$ is a solution of (2.7); hence $\tilde{V}\left(t, X_{t}, Y_{t}\right)$ coincides with the value process $\mathrm{V}$ by Theorem 1 . Therefore $\tilde{V}\left(t, X_{t}, Y_{t}\right)=V\left(t, X_{t}, Y_{t}\right)$ a.s. and $\tilde{V}=V, d t d x d y$ a.e.

It is evident that Theorem 1 and equality (2.8) imply that $\nu^{*}=-\sigma^{\perp^{\prime}} V_{y}$.

Remark. Since the infimum in (3.3) is attained for $\nu^{*}=-\sigma^{\perp^{\prime}} V_{y}$, we can rewrite this equation as

$$
\begin{aligned}
L V(t, x, y)+ & \frac{1}{2}\|\theta(t, x, y)\|^{2} \\
+ & b^{\prime}(t, x, y) V_{y}(t, x, y)-\theta^{\prime}(t, x, y) \delta^{\prime}(t, x, y) V_{y}(t, x, y) \\
& \quad-\frac{1}{2}\left\|\sigma^{\perp^{\prime}}(t, x, y) V_{y}(t, x, y)\right\|^{2}=0 d t d x d y \text {-a.s. }
\end{aligned}
$$

Now we consider two particular cases where equation (3.3) admits an explicit solution.

Case 1. Suppose that the price process $X$ is described by the PDE

$$
d X_{t}=\operatorname{diag}\left(X_{t}\right)\left(\mu\left(t, X_{t}\right) d t+\sigma^{l}\left(t, X_{t}\right) d W_{t}^{l}\right), \quad t \in[0, T],
$$

where $\sigma^{l}$ satisfies the uniform ellipticity condition, $\mu$ and $\sigma^{l}$ are bounded, measurable and such that equation (3.10) admits a unique strong solution. Then

$$
F_{t}^{l}=F_{t}^{X}
$$

and the market price of risk $\theta\left(t, X_{t}\right)$ is $F_{t}^{l}$-measurable.

It is well known (see, e.g., [14]) that in this case the minimal entropy martingale measure coincides with the minimal martingale measure $Q^{\text {min }}$. Let $E^{\text {min }}$ be the mathematical expectation with respect to the minimal martingale measure. By Girsanov's theorem the process

$$
\tilde{W}_{t}^{l}=\int_{0}^{t} \theta\left(s, W^{l}\right) d s+W_{t}^{l}
$$


is a Brownian motion with respect to the measure $Q^{\text {min }}$ and by the integral representation theorem (and the Bayes rule) any $Q^{\mathrm{min}}$-local martingale adapted to $F^{l}$ is represented as a stochastic integral and hence

$$
\int_{0}^{T}\left\|\theta_{s}\right\|^{2} d s=E^{\min } \int_{0}^{T}\left\|\theta_{s}\right\|^{2} d s+\int_{0}^{T} \tilde{\psi}_{s}^{\prime} d \tilde{W}_{s}^{l}
$$

It is easy to verify that the triple $\left(K, \psi, \psi^{\perp}\right)$, where $\psi=\frac{1}{2} \tilde{\psi}, \psi^{\perp}=0$ and

$$
K_{t}=\frac{1}{2} E^{\min }\left(\int_{t}^{T}\left\|\theta_{s}\right\|^{2} d s \mid F_{t}^{l}\right)
$$

characterizes the unique solution of (2.7). Therefore $V_{t}=K_{t}$ and the Markov property of $X$ implies that $V_{t}=V\left(t, X_{t}\right)$ a.s., where

$$
V(t, x)=\frac{1}{2} E^{\min }\left(\int_{t}^{T}\left\|\theta\left(s, X_{s}\right)\right\|^{2} d s / X_{t}=x\right) .
$$

Since the conditions of Theorem 2 are satisfied, $V(t, x)$ is the unique bounded solution of the equation

$$
L V(t, x)+\frac{1}{2}\|\theta(t, x)\|^{2}=0, \quad V(T, x)=0
$$

in the class $V^{L}$.

Under suitable regularity conditions on $\mu$ and $\sigma^{l}$, (see, e.g., [6]), the value function $V(t, x)$ is the unique bounded solution of (3.14) from the class $C^{1,2}$ and

$$
L V=\frac{\partial V}{\partial t}+\frac{1}{2} \operatorname{tr}\left(\operatorname{diag}(x) \sigma^{l} \sigma^{l^{\prime}} \operatorname{diag}(x) V_{x x}\right) .
$$

Case 2. Let us consider the stochastic volatility model

$$
\begin{aligned}
d X_{t} & =\operatorname{diag}\left(X_{t}\right)\left(\mu\left(t, Y_{t}\right) d t+\sigma^{l}\left(t, Y_{t}\right) d W_{t}^{l}\right), \\
d Y_{t} & =b\left(t, Y_{t}\right) d t+\sigma^{\perp}\left(t, Y_{t}\right) d W_{t}^{\perp},
\end{aligned}
$$

where equation (3.15) admits a unique strong solution. We assume that the coefficients of (3.15) satisfy B1) and B2). We have that $F^{\perp}=F^{Y}$ and the market price of risk $\theta\left(t, Y_{t}\right)$ is $F_{t}^{\perp}$ adapted.

Since $\theta$ is $F^{\perp}$ adapted, by the integral representation theorem there exists an $F^{\perp}$ adapted process $g$ such that

$$
\exp \left\{-\frac{1}{2} \int_{0}^{T}\left\|\theta_{s}\right\|^{2} d s\right\}=E \exp \left\{-\frac{1}{2} \int_{0}^{T}\left\|\theta_{s}\right\|^{2} d s\right\}+\int_{0}^{T} g_{s}^{\prime} d W_{s}^{\perp},
$$

where $g \cdot W^{\perp}$ is a bounded martingale.

Using the Itô formula one can see that the triple $\left(K, \psi, \psi^{\perp}\right)$, where

$$
\psi=0, \quad \psi_{t}^{\perp}=-\frac{g_{t}}{E \exp \left\{-\frac{1}{2} \int_{0}^{T}\left\|\theta_{s}\right\|^{2} d s\right\}+\int_{0}^{t} g_{s}^{\prime} d W_{s}^{\perp}}
$$


and

$$
K_{t}=\frac{1}{2} E\left(\int_{t}^{T}\left(\left\|\theta_{s}\right\|^{2}-\left\|\psi_{s}^{\perp}\right\|^{2}\right) d s \mid F_{t}^{\perp}\right)
$$

is the unique solution of $(2.7)$.

Therefore, the process $K$ coincides with the value process $V$ and hence the value function does not depend on $x$, i.e., $V(t, x, y)=V(t, y)$. Thus, by Theorem $2, V(t, y)$ is the unique bounded solution in the class $V^{L}$ of the equation

$$
\begin{gathered}
L V(t, y)+\frac{1}{2}\|\theta(t, y)\|^{2}+V_{y}^{\prime}(t, y) b(t, y) \\
-\frac{1}{2}\left\|\sigma^{\perp^{\prime}}(t, y) V_{y}(t, y)\right\|^{2}=0 \quad d t d y \text {-a.s } \\
V(T, y)=0 .
\end{gathered}
$$

For $U(t, y)=e^{-V(t, y)}$ equation (3.17) can be reduced to a linear SDE

$$
L U(t, y)+b^{\prime}(t, y) U_{y}(t, y)+\frac{1}{2}\|\theta(t, y)\|^{2} U(t, y)=0 .
$$

Under additional smoothness conditions on the coefficients $\mu, \sigma^{l}, b$ and $\sigma^{\perp}$ (see [6]) this equation with the boundary condition $U(T, y)=1$ has the unique solution in the class $C^{1,2}$ with $L V$ being the usual $L$-operator. By the FeynmannKac formula the value admits a representation

$$
V(t, y)=-\ln E\left[\exp \left\{\frac{1}{2} \int_{t}^{T}\left|\theta\left(s, Y_{s}\right)\right|^{2} d s \mid\right\} \mid Y_{t}=y\right]
$$

Remark. The solvability of (3.3) (or (3.9)) in the class $C^{1,2}$ is apparently an open problem. The value function can be characterized as a viscosity solution of (3.3), e.g., using the results from [3]. we solve equation (3.3) in the class $V^{L}$ of functions which, unlike viscosity solutions, admit all generalized first order derivatives at $x$.

\section{ApPEndix}

The appendix is devoted to the introduction of some notions which enable us to present an application of Theorem 1 to the Markov case.

Consider the system of stochastic differential equations (3.1), (3.2) and assume that conditions B1) and B2) are satisfied. Under these conditions there exists a unique weak solution of (3.1), (3.2), which is a Markov process and its transition probability function admits a density $p\left(s,\left(x_{0}, y_{0}\right), t,(x, y)\right)$ with respect to the Lebesgue measure. We shall use the notation $p(t, x, y)=p\left(0,\left(x_{0}, y_{0}\right), t,(x, y)\right)$ for the fixed initial condition $X_{0}=x_{0}, Y_{0}=y_{0}$.

Introduce the measure $\mu$ on the space $\left([0, T] \times R_{+}^{d} \times R^{n-d}, \mathcal{B}\left([0, T] \times R_{+}^{d} \times\right.\right.$ $\left.\left.R^{n-d}\right)\right)$

$$
\mu(d s, d x, d y)=p(s, x, y) d s d x d y
$$


Let $C^{1,2}$ be the class of functions $f$ continuously differentiable at $t$ and twice differentiable at $x, y$ on $[0, T] \times R_{+}^{d} \times R^{n-d}$. For functions $f \in C^{1,2}$ the $L$ operator is defined as

$$
\begin{aligned}
L f= & f_{t}+\operatorname{tr}\left(\frac{1}{2} \operatorname{diag}(x) \sigma^{l} \sigma^{l^{\prime}} \operatorname{diag}(x) f_{x x}\right) \\
& +\operatorname{tr}\left(\delta \sigma^{l^{\prime}} \operatorname{diag}(x) f_{x y}\right)+\operatorname{tr}\left(\frac{1}{2}\left(\delta \delta^{\prime}+\sigma^{\perp}{\sigma^{\perp^{\prime}}}^{\prime}\right) f_{y y}\right)
\end{aligned}
$$

where $f_{t}, f_{x x}, f_{x y}$ and $f_{y y}$ are partial derivatives of the function $f$, for which we use the matrix notations.

Definition A. We shall say that a function $f=\left(f(t, x, y), t \geq 0, x \in R_{+}^{d}, y \in\right.$ $\left.R^{n-d}\right)$ belongs to the class $V_{\mu}^{L}$ if there exists a sequence of functions $\left(f^{n}, n \geq 1\right)$ from $C^{1,2}$ and measurable $\mu$-integrable functions $f_{x_{i}}(i \leq d), f_{y_{j}}(d<j \leq n)$ and $(L f)$ such that

$$
\begin{aligned}
& E \sup _{s \leq T}\left|f^{n}\left(s, X_{s}, Y_{s}\right)-f\left(s, X_{s}, Y_{s}\right)\right| \rightarrow 0, \quad \text { as } n \rightarrow \infty, \\
& \quad \iint_{[0, T] \times R_{+}^{d} \times R^{n-d}}\left(f_{x_{i}}^{n}(s, x, y)-f_{x_{i}}(s, x, y)\right)^{2} x_{i}^{2} \mu(d s, d x, d y) \rightarrow 0, \quad \text { as } n \rightarrow \infty, \quad i \leq d,
\end{aligned}
$$

$\iint_{[0, T] \times R_{+}^{d} \times R^{n-d}}\left(f_{y_{j}}^{n}(s, x, y)-f_{y_{j}}(s, x, y)\right)^{2} \mu(d s, d x, d y) \rightarrow 0, \quad$ as $n \rightarrow \infty, \quad d<j \leq n$, $\iint_{[0, T] \times R_{+}^{d} \times R^{n-d}}\left|L f^{n}(s, x, y)-(L f)(s, x, y)\right| \mu(d s, d x, d y) \rightarrow 0, \quad$ as $n \rightarrow \infty$.

Now we formulate the statement proved in [2] in the case convenient for our purposes.

Proposition A. Let conditions $\mathrm{B} 1)-\mathrm{B} 2)$ be satisfied and let $f\left(t, X_{t}, Y_{t}\right)$ be a bounded process. Then the process $\left(f\left(t, X_{t}, Y_{t}\right), t \in[0, T]\right)$ is an Itô process of the form

$$
f\left(t, X_{t}, Y_{t}\right)=f\left(0, X_{0}, Y_{0}\right)+\int_{0}^{t} g(s, \omega) d W_{s}+\int_{0}^{t} a(s, \omega) d s \quad a . s .
$$

with

$$
E \int_{0}^{t} g^{2}(s, \omega) d s<\infty, E \int_{0}^{t}|a(s, \omega)| d s<\infty
$$

if and only if $f$ belongs to $V_{\mu}^{L}$. Moreover the process $f\left(t, X_{t}, Y_{t}\right)$ admits the decomposition

$$
f\left(t, X_{t}, Y_{t}\right)=f\left(0, X_{0}, Y_{0}\right)+\sum_{i=1}^{d} \int_{0}^{t} f_{x_{i}}\left(s, X_{s}, Y_{s}\right) d X_{s}^{i}
$$




$$
+\sum_{j=d+1}^{n} \int_{0}^{t} f_{y_{j}}\left(s, X_{s}, Y_{s}\right) d Y_{s}^{j}+\int_{0}^{t}(L f)\left(s, X_{s}, Y_{s}\right) d s
$$

Remark. For continuous functions $f \in V_{\mu}^{L}$ the condition

$$
\sup _{(s, x, y) \in D}\left|f^{n}(s, x, y)-f(s, x, y)\right| \rightarrow 0 \text { as } n \rightarrow \infty
$$

for every compact $D \in[0, T] \times R_{+}^{d} \times R^{n-d}$, can be used instead of the first relation of Definition A.

\section{REFERENCES}

1. F. Biagini, P. Guasoni and M. Pratelli, Mean-variance hedging for stochastic volatility models. INFORMS Applied Probability Conference (Ulm, 1999). Math. Finance 10(2000), No. 2, 109-123.

2. R. Chitashvili and M. Mania, Generalized Itô formula and derivation of Bellman's equation. Stochastic processes and related topics (Siegmundsberg, 1994), 1-21, Stochastics Monogr., 10, Gordon and Breach, Yverdon, 1996.

3. M. G. Crandall, H. Ishi and P. L. Lions, User's guide to viscosity solutions of second order partial differential equations. Bull. Amer. Math. Soc. (N.S.) 27(1992), No. $1,1-67$.

4. F. Delbaen, P. Grandits, Th. Rheinländer, D. Samperi, W. Schweizer, and Ch. Stricker, Exponential hedging and entropic penalties. Math. Finance 12(2002), No. 2, 99-123.

5. N. El Karoui, S. Peng And M. C. Quenez, Backward stochastic differential equations in finance. Math. Finance 7(1997), No. 1, 1-71.

6. A. Friedman, Stochastic differential equations and applications. Vol. 1. Probability and Mathematical Statistics, 28. Academic Press [Harcourt Brace Jovanovich, Publishers], New York-London, 1975.

7. M. FRITtelli, The minimal entropy martingale measure and the valuation problem in incomplete markets. Math. Finance 10 (2000), No. 1, 39-52.

8. P. Grandits and T. Rheinländer, On the minimal entropy martingale measure. Ann. Probab. 30(2002), No. 3, 1003-1038.

9. I. Karatzas, J. Lehoczky, S. Schreve, and G. L. Xu, Martingale and duality methods for utility maximization in an incomplete market. SIAM J. Control Optim. 29(1991), No. 3, 702-730.

10. N. Kazamaki, Continuous exponential martingales and BMO. Lecture Notes in Mathematics, 1579. Springer-Verlag, Berlin, 1994.

11. J. P. Laurent and H. Pham, Dynamic programming and mean-variance hedging. Finance Stoch. 3(1999), No. 1, 83-110.

12. M. Mania, M. Santacroce, and R. Tevzadze, A semimartingale BSDE related to the minimal entropy martingale measure. Finance Stoch. $\mathbf{7}$ (2003), No. 3, 385-402.

13. Y. Miyahara, Canonical martingale measures of incomplete assets markets. Probability theory and mathematical statistics (Tokyo, 1995), 343-352, World Sci. Publishing, River Edge, NJ, 1996.

14. H. Pham, T. Rheinländer, And M. Schweizer, Mean-variance hedging for continuous processes: new proofs and examples. Finance Stoch. 2(1998), No. 2, 173-198. 
15. R. Rouge and N. El Karoui, Pricing via utility maximization and entropy. INFORMS Applied Probability Conference (Ulm, 1999). Math. Finance 10(2000), No. 2, 259-276.

16. M. SchweIzer, Martingale densities for general asset prices. J. Math. Econom. 21(1992), No. $4,363-378$.

(Received 14.09.2003)

Authors' addresses:

Michael Mania

A. Razmadze Mathematical Institute

Georgian Academy of Sciences

1, M. Aleksidze St., Tbilisi 0193

Georgia

E-mail: mania@rmi.acnet.ge

Marina Santacroce

Dipartimento di Matematica

Politecnico di Torino

Italia

Revaz Tevzadze

Institute of Cybernetics

Georgian Academy of Sciences

1, S. Euli St., Tbilisi 0186

Georgia 